\title{
Effect of Evaporation Losses on Experimental Continuous Culture Results
}

\author{
By W. R. KING, C. G. SINCLAIR AND H. H. TOPIWALA \\ Department of Chemical Engineering, University of Manchester Institute \\ of Science and Technology, Manchester
}

(Accepted for publication 8 January 1972)

SUMMARY

Evaporation can lead to errors in the measurement of parameters such as yield and maintenance coefficient, using continuous cultures at dilution rates below $0.05 \mathrm{~h}^{-1}$.

\section{INTRODUCTION}

This paper shows how simple practical details, if ignored, can lead to false conclusions regarding the kinetic model used for a continuous culture system. The need to humidify the air, when operating a continuous culture system at low dilution rates, can be assessed by measuring the function $\phi=\bar{x}_{\mathrm{e}} / \bar{x}$, where $\bar{x}_{\mathrm{e}}$ and $\bar{x}$ are the cell concentrations, which would be observed for evaporating and non-evaporating conditions, respectively. As shown later, this function becomes significant at dilution rates below $0.05 \mathrm{~h}^{-1}$, values which were used in work reported for example by Marr, Nilson \& Clark (1963) to calculate a maintenance energy constant (a), by Schulze \& Lipe (1964) and by Tempest, Herbert \& Phipps (1967). This error can be avoided by fixing a condenser on the gas outlet of the fermenter.

The rate of evaporation, $D_{2}$, will be a function of several parameters, including the stirrer speed, the temperature and humidity of the inlet air, the temperature of the liquid medium, the flow rate of air and the design of the vessel. The effect of evaporation has been calculated for three different theoretical models (Fig. 2 and 3).

\section{THEORY}

In Fig. I, $F_{0}$ is the inlet liquid flow rate, $F_{1}$ the exit liquid flow rate and $F_{2}$ the evaporation rate of water into the air stream, all in units of litres of liquid per hour. The basic assumptions of a completely stirred tank reactor (C.S.T.R.) are assumed to hold for the models developed (i.e. the organism and substrate concentrations in the vessel are completely homogeneous and equal to their exit concentrations).

Monod's model. This is based on Monod's model (Monod, 1942, 1950) for the growth rate, $\mu$.

Organism balance:

$$
V \frac{\mathrm{d} x_{\mathrm{e}}}{\mathrm{d} t}=\frac{V \mu_{\mathrm{m}} x_{\mathrm{e}} S_{\mathrm{e}}}{K_{\mathrm{s}}+S_{\mathrm{e}}}-F_{1} x_{\mathrm{e}}
$$

Substrate balance:

$$
\text { Accumulation }=\text { Input }- \text { Output. }
$$

Overall balance:

$$
V \frac{\mathrm{d} S_{\mathrm{e}}}{\mathrm{d} t}=F_{0} S_{\mathrm{R}}-F_{1} S_{\mathrm{e}}-\frac{V \mu_{\mathrm{m}} x_{\mathrm{e}} S_{\mathrm{e}}}{Y\left(K_{\mathrm{s}}+S_{\mathrm{e}}\right)}
$$

$$
F_{0}=F_{1}+F_{2} \text {. }
$$


At steady state, $\mathrm{d} x_{\mathrm{e}} / \mathrm{d} t=\mathrm{d} S_{\mathrm{e}} / \mathrm{d} t=0$.

From equation (I):

From equation (2):

$$
0=\frac{\mu_{\mathrm{m}} \bar{x}_{\mathrm{e}} \bar{S}_{\mathrm{e}}}{K_{\mathrm{s}}+\bar{S}_{\mathrm{e}}}-D_{1} \bar{x}_{\mathrm{e}}
$$

From equation (3):

$$
0=D_{0} S_{\mathrm{R}}-D_{1} \bar{S}_{\mathrm{e}}-\frac{\mu_{\mathrm{m}} \bar{x}_{\mathrm{e}} \bar{S}_{\mathrm{e}}}{Y\left(K_{\mathrm{S}}+\bar{S}_{\mathrm{e}}\right)}
$$

$$
D_{1}=D_{0}-D_{2}
$$

where $D_{1}=F_{1} / V$, therefore $\bar{S}_{\mathrm{e}}=K_{\mathrm{s}} D_{1} /\left(\mu_{\mathrm{m}}-D_{1}\right)$ and $\bar{x}_{\mathrm{e}}=Y / D_{1}\left(D_{0} S_{\mathrm{R}}-D_{1} \bar{S}_{\mathrm{e}}\right)$.

If $D_{0}=D_{1}, \bar{x}=Y\left(S_{\mathrm{R}}-\bar{S}\right)$, giving the steady-state cell concentration of the normal Monod model without evaporation.

Therefore, the expression for the parameter, $\phi$, becomes

$$
\phi=\frac{\bar{x}_{\mathrm{e}}}{\bar{x}}=\frac{D_{0} S_{\mathrm{R}}-D_{1} \bar{S}_{\mathrm{e}}}{D_{1}\left(S_{\mathrm{R}}-\bar{S}\right)}
$$

At low values of $D, \bar{S}$ and $\bar{S}_{\mathrm{e}} \ll S_{\mathrm{R}}$, therefore $\phi \approx D_{0} / D_{1}=D_{0} /\left(D_{0}-D_{2}\right)$.

Endogenous model. The second model is based on Herbert's formulation of endogenous metabolism (Herbert, I958), in which the constant, $k$, accounts for the oxidation of cell substance to $\mathrm{CO}_{2}$. This constant endogenous metabolism added to the anabolic metabolism of the culture gives the modified equation set for evaporation.

Organism balance:

$$
V \frac{\mathrm{d} x_{\mathrm{e}}}{\mathrm{d} t}=V\left[\frac{\left(\mu_{\mathrm{m}}+k\right) S_{\mathrm{e}}}{K_{\mathrm{s}}+S_{\mathrm{e}}}-k\right] x_{\mathrm{e}}-F_{1} x_{\mathrm{e}}
$$

Substrate balance:

$$
V \frac{\mathrm{d} S_{\mathrm{e}}}{\mathrm{d} t}=F_{0} S_{\mathrm{R}}-F_{1} S_{\mathrm{e}}-\frac{V\left(\mu_{\mathrm{m}}+k\right) x_{\mathrm{e}} S_{\mathrm{e}}}{Y_{\mathrm{p}}\left(K_{\mathrm{s}}+S_{\mathrm{e}}\right)} .
$$

As before, the steady-state equations are solved to give:

$$
\begin{aligned}
& \bar{S}_{\mathrm{e}}=\frac{K_{\mathrm{s}}\left(D_{1}+k\right)}{\mu_{\mathrm{m}}-D_{1}}, \\
& \bar{x}_{\mathrm{e}}=\frac{Y_{\mathrm{p}}\left(D_{0} S_{\mathrm{R}}-D_{1} \bar{S}_{\mathrm{e}}\right)}{D_{1}+k}, \\
& D_{0}=D_{1}+D_{2} .
\end{aligned}
$$

For $D_{0}=D_{1}$, we obtain the cell concentration under non-evaporating conditions.

$$
\vec{x}=\frac{Y_{\mathrm{p}} D_{0}\left(S_{\mathrm{R}}-\bar{S}\right)}{D_{0}+k} .
$$

Therefore

$$
\frac{\bar{x}_{\mathrm{e}}}{\bar{x}}=\frac{D_{0} S_{\mathrm{R}}-D_{1} \bar{S}_{\mathrm{e}}}{\left(D_{1}+k\right)\left(S_{\mathrm{R}}-\bar{S}\right)} \frac{D_{0}+k}{D_{0}}
$$

and at low dilution rates, $\phi \approx\left(D_{0}+k\right) /\left(D_{0}+k-D_{2}\right)$.

Maintenance model. This was suggested by Pirt (1965), who included a specific maintenance term (a) accounting for the consumption of the limiting substrate to provide energy to maintain integrity and viability, but no biomass.

This maintenance model, when modified for evaporation, gives the equation set.

Organism balance:

$$
\frac{\mathrm{d} x_{\mathrm{e}}}{\mathrm{d} t}=\frac{\mu_{\mathrm{m}} S_{\mathrm{e}}}{K_{\mathrm{s}}+S_{\mathrm{e}}} x_{\mathrm{e}}-D_{1} x_{\mathrm{e}}
$$




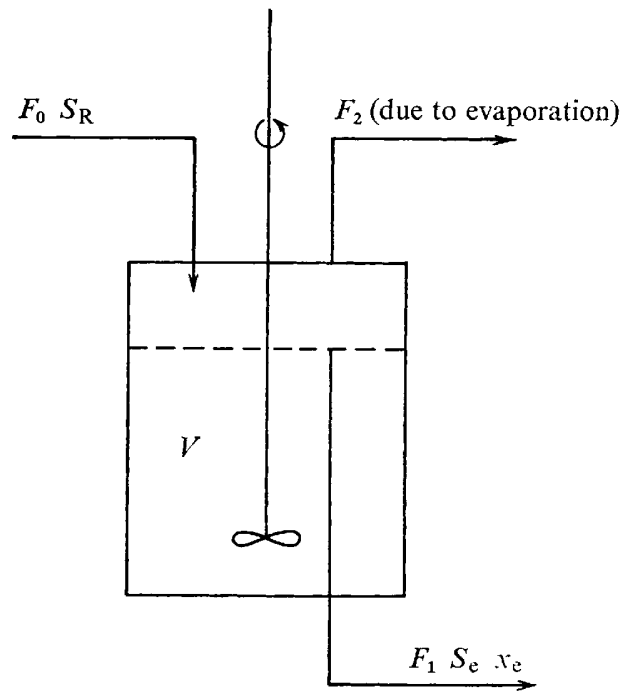

Fig. I. Evaporating model.

Table I.

Type and reference

Continuous culture (Pirt, 1957)

Continuous culture (this work)

Continuous culture (this work) $250 \mathrm{ml}$ shake flask with $100 \mathrm{ml}$ of culture (this work)
Air flow rate

(l liquid/h)

Temp.

$\left({ }^{\circ} \mathrm{C}\right)$

Agitator speed (rev./min)

1030

250

450

I 25
$D_{2}$
$\left(\mathrm{~h}^{-1}\right)$ 0.003

0.0013

0.00275

0.00034

Substrate balance:

$$
\frac{\mathrm{d} S_{\mathrm{e}}}{\mathrm{d} t}=D_{0} S_{\mathrm{R}}-D_{1} S_{\mathrm{e}}-\frac{\mu_{\mathrm{m}} x_{\mathrm{e}} S_{\mathrm{e}}}{Y_{\mathrm{g}}\left(K_{\mathrm{s}}+S_{\mathrm{e}}\right)}-\frac{a x_{\mathrm{e}}}{Y_{\mathrm{g}}} .
$$

As before, the steady-state equations are solved to give

$$
\begin{aligned}
\bar{S}_{\mathrm{e}} & =\frac{K_{\mathrm{s}} D_{1}}{\mu_{\mathrm{m}}-D_{1}}, \\
\vec{x}_{\mathrm{e}} & =\frac{Y_{\mathrm{g}}\left(D_{0} S_{\mathrm{R}}-D_{1} \bar{S}_{\mathrm{e}}\right)}{D_{1}+a}, \\
D_{0} & =D_{1}+D_{2} .
\end{aligned}
$$

For non-evaporating conditions,

and therefore

$$
\bar{x}=\frac{Y_{\mathrm{g}} D_{0}}{D_{0}+a}\left(S_{\mathrm{R}}-\bar{S}\right)
$$

$$
\phi=\frac{\bar{x}_{\mathrm{e}}}{\bar{x}}=\frac{D_{0} S_{\mathrm{R}}-D_{1} \bar{S}_{\mathrm{e}}}{\left(D_{1}+a\right)\left(S_{\mathrm{R}}-\bar{S}\right)} \frac{D_{0}+a}{D_{0}}
$$

and at low dilution rates, $\phi=\left(D_{0}+a\right) /\left(D_{0}+a-D_{2}\right)$, which is identical in form to the simplified expression for the endogenous metabolism models. 


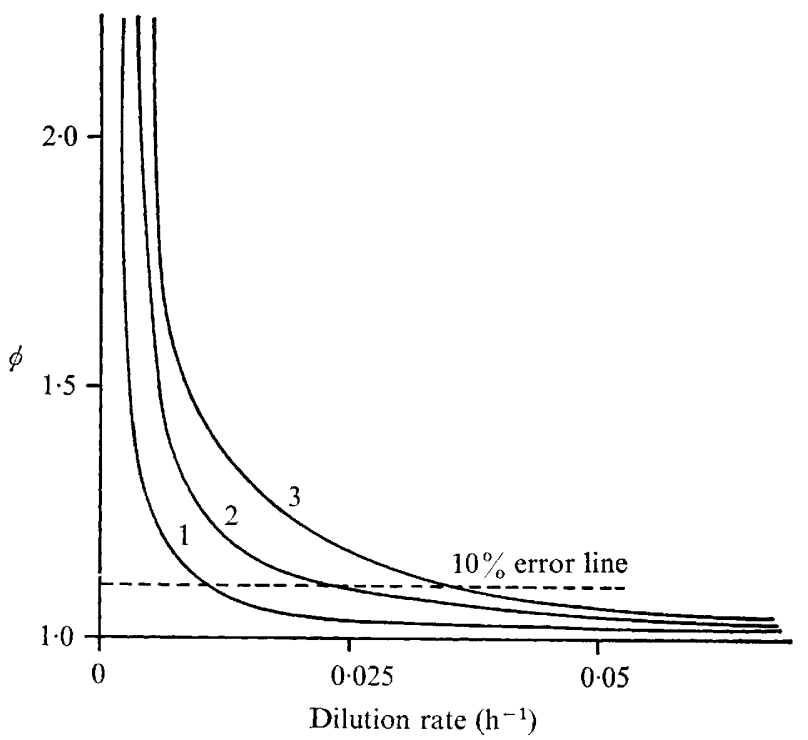

Fig. 2. Monod's model errors. Curve 1, $D_{2}=0.001$; curve 2, $D_{2}=0.002 ;$ curve $3, D_{2}=0.003$.

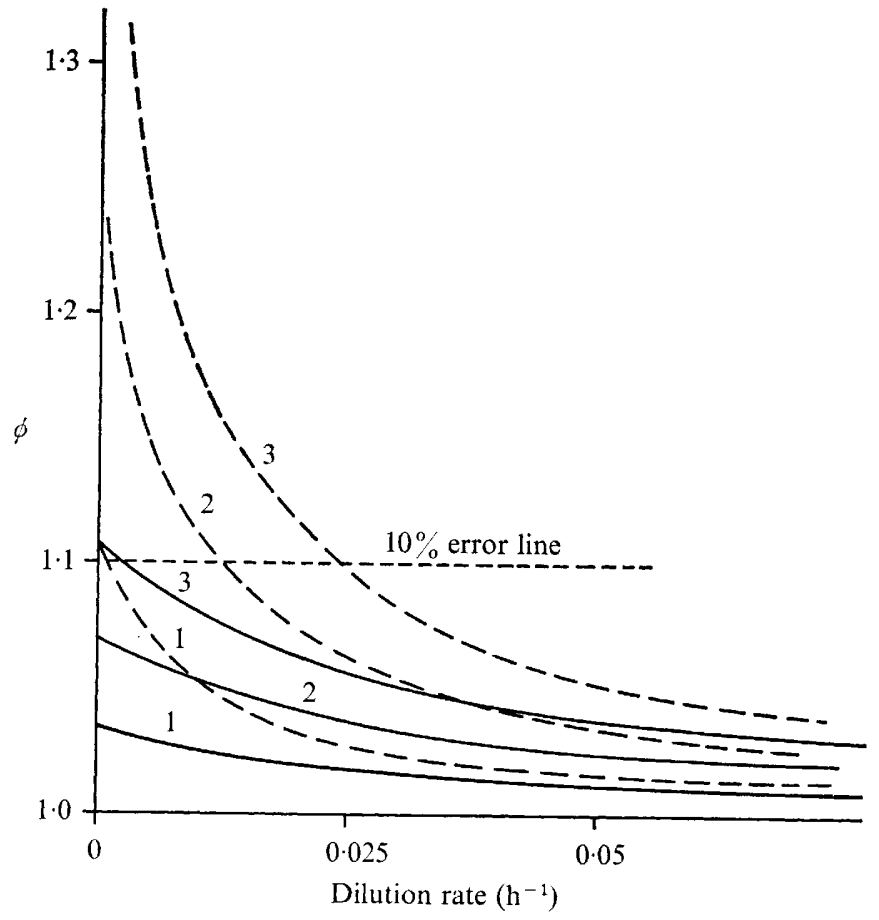

Fig. 3. Endogenous metabolism or maintenance model errors. _-, $k$ (or $a)=0.03$; - - - , $k$ or $(a)=0.01 ;$ curve I, $D_{2}=0.001$; curve $2, D_{2}=0.002 ;$ curve $3, D_{2}=0.003$. 


\section{EXPERIMENTAL}

Realistic values of the parameters in these expressions for $\phi$ may be obtained from the literature, except for the effective evaporation rate $\left(D_{2}\right)$. Pirt (I957) has published a value for evaporation in vigorously agitated laboratory-scale continuous culture. This is included together with some experimental values for $D_{2}$ obtained by us (Table I).

The parameter, $\phi$, was calculated from the simplified expression derived in the theory section and plotted against $D_{0}$ for a practical range of $D_{2}$, as shown in Fig. 2 and 3. The expression for $\phi$ is identical for the endogenous and maintenance models and therefore the effect of different values of ' $k$ ' and ' $a$ ', respectively, will be identical.

\section{DISCUSSION}

The figures indicate that, for negligible endogenous metabolism, the accepted formulae connecting $D, \bar{x}_{\mathrm{e}}$ and $\bar{S}_{\mathrm{e}}$ need modification at low dilution rates and Herbert's model should read,

$$
\begin{aligned}
\bar{x}_{\mathrm{e}} & =Y_{\mathrm{p}} \frac{D_{0} S_{\mathrm{R}}-D_{\mathrm{I}} \bar{S}_{\mathrm{e}}}{D_{1}+k} \\
& =Y_{\mathrm{p}} \frac{D_{1}}{D_{1}+k}\left(\frac{D_{0}}{D_{1}} S_{\mathrm{R}}-\bar{S}_{\mathrm{e}}\right) .
\end{aligned}
$$

The culture behaves exactly as if the dilution rate were $D_{1}$, as indeed it is as far as the organism is concerned, with the ingoing substrate concentration of $D_{0} S_{\mathrm{R}} / D_{1}$. Even where the endogenous metabolism or maintenance energy constant is relatively large, the errors are not negligible. Steady state can be obtained at dilution rates down to $0.004 \mathrm{~h}^{-1}$ (Postgate \& Hunter, 1962; Tempest et al. 1967) in bacterial cultures and that fungal cultures are operated at dilution rates as low as $0.0025 \mathrm{~h}^{-1}$ (Holme \& Zacharias, I965) with an endogenous metabolism constant $k$ of nearly zero.

The low value for evaporation in shake flasks (Table I; $D_{2}=0.0034 \mathrm{~h}^{-1}$ ) must be considered in the context of the period of time the shake flask is on the rotary incubator. At this rate of evaporation the error in the measurement of organism concentration rises linearly with time and would be approximately $4 \%$ after five days.

We conclude therefore that errors in organism concentrations at low dilution rates can be significant especially for systems with low endogenous metabolism rates, when no special precautions are taken to reduce evaporation losses. When evaluating the endogenous metabolism constant $(k)$ by plotting $\frac{I}{x}$ versus $\frac{\mathrm{I}}{D_{0}}$, the slope of the line is equal to $\left(k-D_{2}\right) / Y_{\mathrm{p}} S_{\mathrm{R}}$ and not $k / Y_{\mathrm{p}} S_{\mathrm{R}}$. Thus, if evaporation were not taken into account the value of ' $k$ ' (or ' $a$ ') would be underestimated by an amount $D_{2} \mathrm{~h}^{-1}$.

\section{Symbols}

a Substrate utilization constant due to maintenance requirements $\mathrm{h}^{-1}$

$D \quad$ Dilution rate

$h^{-1}$

$F_{0} \quad$ Inlet liquid flow rate

$1 / \mathrm{h}$

$F_{1} \quad$ Exit liquid flow rate

$1 / \mathrm{h}$

$F_{2} \quad$ Evaporation rate of water

$1 / \mathrm{h}$

$k$ Substrate utilization constant due to endogenous metabolism

$\mathrm{h}^{-1}$

$K_{8} \quad$ Monod's substrate constant, numerically equal to $S$ at which the rate is $\frac{1}{2} \mu_{\mathrm{m}}$

$S \quad$ Substrate concentration under non-evaporating conditions

$\mathrm{g} / 1$

$\mathrm{g} / 1$ 
$S_{\mathrm{e}} \quad$ Substrate concentration under evaporating conditions $\mathrm{g} / \mathbf{l}$

$S_{\mathrm{R}} \quad$ Inlet substrate concentration $\quad \mathrm{g} / \mathrm{l}$

$t$ Time $\mathrm{h}$

$x \quad$ Organism concentration under non-evaporating conditions $\mathrm{g} / \mathrm{l}$

$x_{\mathrm{e}} \quad$ Organism concentration under evaporating conditions g/l

$Y$ Potential yield constant for the Monod model dimensionless

$Y_{\mathrm{p}} \quad$ Potential yield constant for the endogenous metabolism model dimensionless

$Y_{\mathrm{g}} \quad$ Potential yield constant for the maintenance model dimensionless

$\mu(S)$ Specific growth rate $\mathrm{h}^{-1}$

$\mu_{\mathrm{m}} \quad$ Maximum growth rate $\mathrm{h}^{-1}$

$\phi \quad=\bar{x}_{\mathrm{e}} / \bar{x}$

$\left(^{-}\right) \quad$ Indicates steady-state values

\section{REFERENCES}

Herbert, D. (1958). Some principles of continuous culture. In Recent Progress in Microbiology. Symposium VII of the International Congress of Microbiology, p. 381. Stockholm: Almquist \& Wiksell.

Holme, F. \& ZACHARIAS, B. (I965). Giberellic acid formation in continuous culture. Biotechnology \& Bioengineering 7, 405-415.

Marr, A. G., Nilson, E. H. \& Clark, D. J. (I963). The maintenance requirement of Escherichia coli. Annals of the New York Academy of Science ro2, 536-548.

MonoD, J. (1942). Recherches sur la croissance des cultures bactériennes. Paris: Hermann \& Cie.

Monod, J. (I950). La technique de culture continue; theorie et applications. Annales de l'Institut Pasteur 79, $390-410$.

PIRT, S. J. (1957). The oxygen requirement of growing cultures of an Aerobacter species determined by means of the continuous culture technique. Journal of General Microbiology 16, 59-75.

PIRT, S. J. (1965). The maintenance energy of bacteria in growing cultures. Proceedings of Royal Society B r65, 224-23I.

Postgate, J. R. \& Hunter, J. R. (I962). The survival of starved bacteria. Journal of General Microbiology 29. 233-263.

SCHUlZE, K. \& LIPE, R. S. (1964). Relationship between substrate concentration, growth rate and respiration rate of Escherichia coli in continuous culture. Archiv für Mikrobiologie 48, I-20.

Tempest, D. W., Herbert, D. \& Phipps, P. J. (1967). Studies on the growth of Aerobacter aerogenes at low dilution rates in a chemostat. In Microbial Physiology and Continuous Culture. London: Her Majesty's Stationery Office. 